\title{
Verrucous Oesophageal Carcinoma: Single Case Report and Case Series Including 15 Patients - Issues for Consideration of Therapeutic Strategies
}

\author{
Angelika Behrens $^{\mathrm{a}} \quad$ Manfred Stolte $^{\mathrm{b}} \quad$ Oliver Pech $^{\mathrm{c}} \quad$ Andrea May $^{\mathrm{d}}$ Christian Ell $^{\mathrm{d}}$ \\ a Internal Medicine, Vivantes Klinikum im Friedrichshain, University Teaching Hospital of the Humboldt University Berlin (Charité), \\ Berlin, Germany, \\ bInstitute of Pathology, Kulmbach Hospital, Kulmbach, Germany, \\ cInternal Medicine, St. John of God Hospital, Regensburg, Germany, \\ dInternal Medicine II, Sana Klinikum Offenbach, Offenbach, Germany
}

\section{Keywords}

Verrucous oesophageal carcinoma - Endoscopic therapy .

Therapeutic strategy $\cdot$ Case series

\section{Summary}

Background: Verrucous carcinomas (VC) of the oesophagus are a rarity. Due to their histological resemblance to squamous cell carcinoma, the diagnostic and treatment standards applicable to the latter have so far also been applied to $\mathrm{VC}$ as a disease entity. Quite limited data are available including two case series of 5 or 11 patients. The present study reports on a single case treated by local endoscopic therapy and a series of 15 patients, 9 of whom received local endoscopic therapy. Methods: The data for patients diagnosed with VC of the oesophagus who had been treated from January 1999 to May 2011 were analysed retrospectively. Results: 15 patients with the diagnosis of oesophageal VC were included. The male-female ratio was 3:1. 9 of 11 pT1-VC patients presented with the cardinal symptom dysphagia or odynophagia. For the majority of the patients, the growth pattern is one of extensive superficial expansion showing a median length of $9 \mathrm{~cm}$ (range: 2-22 cm). Surprisingly, none of the VC patients showed lymph node or distant metastasis. 9 of $15 \mathrm{VC}$ patients received local endoscopic therapy; 4 were treated with curative intent and 5 were treated palliatively. 3 patients underwent oesophageal resection, and definitive chemoradiotherapy was administered in a further 3 patients. One severe complication, consisting of a postoperative anastomotic insufficiency with a fatal outcome, occurred in this group of patients. Conclusion: This is the largest published study describing patients diagnosed with VC of the oesophagus so far. The option of local endoscopic therapy and its results in 9 patients are reported for the first time. The superficial growth pattern of the tumour and the frequent absence of lymph node or distant metastasis suggest that endoscopic resection can be carried out as a diagnostic and/or therapeutic approach. Due to the rarity of this entity, the case numbers are unfortunately so limited that evidence-based recommendations are unlikely to become available even in the future.

\section{Schlüsselwörte}

Verruköses Ösophaguskarzinom - Endoskopische Therapie . Therapiestrategie · Fallserie

\section{Zusammenfassung}

Hintergrund: Verruköse Ösophaguskarzinome sind eine Rarität. Aufgrund der feingeweblichen Verwandtschaft zum Plattenepithelkarzinom wurden bisher die hierfür gültigen Diagnostikund Therapiestandards auf die Entität der verrukösen Karzinome übertragen. Die Datenlage ist sehr begrenzt und beinhaltet zwei Fallserien mit 5 bzw. 11 Patienten. Methoden: Es wurden alle Patienten eingeschlossen, die im Zeitraum von Januar 1999 bis Mai 2011 aufgrund der Diagnose eines verrukösen Ösophaguskarzinoms behandelt wurden. Ergebnisse: 15 Patienten mit der Diagnose eines verrukösen Ösophaguskarzinoms wurden erfasst. Der überwiegende Anteil der Tumoren zeigte ein oberflächlich nach lateral infiltrierendes Wachstum mit einer medianen Länge von $9 \mathrm{~cm}$ (Bereich: 2-22 cm). Überraschenderweise zeigte das Staging bei keinem der Patienten eine Lymphknoten- oder Fernmetastasierung. 9 der 15 Patienten erhielten eine endoskopische Therapie. 3 Patienten unterzogen sich einer Ösophagusresektion, und weitere 3 Patienten erhielten eine kombinierte Radio-/Chemotherapie. In dieser Gruppe trat als schwere Komplikation eine Anastomoseninsuffizienz auf. Schlussfolgerung: Die vorgelegte Arbeit ist die größte bisher publizierte Serie von Patienten mit der Diagnose eines verrukösen Ösophaguskarzinoms. Erstmalig werden die Möglichkeit sowie die Ergebnisse einer lokalen endoskopischen Therapie bei 9 Patienten berichtet. Das oberflächliche Wachstumsmuster der Tumoren und die Abwesenheit von Lymphknoten- und Fernmetastasen lassen den Tumor für eine endoskopische Therapie geeignet erscheinen. Aufgrund der Seltenheit der Diagnose ist die Fallzahl jedoch so begrenzt, dass auch in Zukunft nicht auf evidenzbasierte Empfehlungen zurückgegriffen werden kann.

\section{KARGER} (C) 2014 S. Karger GmbH, Freiburg $1662-6664 / 14 / 0305-0346 \$ 39.50 / 0$ 


\section{Introduction}

Verrucous carcinomas of the oesophagus are a very rare histological variant of squamous cell carcinoma. In the majority of cases, the lesions are tumours that have a verrucous endoscopic appearance or neoplasias with a papillary surface. In accordance with the World Health Organisation (WHO) classification, the diagnosis is established histologically when, in addition to highly differentiated keratinocytes with minimal cellular atypia, gross cones and space-occupying, non-infiltrative growth in the marginal area are seen [1]. Hyperkeratosis or parakeratosis are often diagnosed, which is further complicated by mycoses. Frequently, time delays in diagnosis occur. In many cases, reliable diagnosis requires a close communication with the specialized gastrointestinal pathologist concerning the suspected diagnosis, repeated biopsy sampling, and, where appropriate, a diagnostic endoscopic resection.

In addition to the classic risk factors of nicotine and alcohol consumption, risk factors for the development of oesophageal verrucous carcinoma mentioned in the literature include chronic oesophagitis (without more detailed definition), achalasia, oesophageal diverticular disease, and gastro-oesophageal reflux disease [2]. The extent to which human papillomavirus (HPV) may play an aetiological role is not known [3].

Apart from the oesophagus, verrucous carcinomas also occur in the ear, nose, and throat (ENT) region, in the genitals, urinary bladder, and anus, as well as on the skin. They are also known as Ackermann tumours after the author of the initial description [4-6].

Quite limited data concerning oesophageal verrucous carcinoma are available, i.e. two series. One was reported by the Mayo Clinic in 1967 and included 5 patients, while the other one, currently available as an abstract, is from 2013 and gives an overview of 11 patients [7, 8]. In addition, approximately
25 individual case reports have been published. In 2000, Tajiri et al. [9] presented the only case report published so far on endoscopic resection of a verrucous carcinoma of the oesophagus.

The present study reports on patients diagnosed with verrucous carcinoma over a period of 12 years: a single case report treated by local endoscopic therapy and a case series of 15 patients, 9 of whom received local endoscopic therapy. Therefore, it is the largest series published worldwide so far. These data provide information about considerable differences between oesophageal verrucous carcinoma and squamous cell carcinoma regarding certain tumour-related characteristics, predisposing comorbidities, and prognosis. In conclusion, it raises the question whether therapy should be individualised.

\section{Patients and Methods}

This retrospective analysis includes a case series of patients who presented at our institution in the period from January 1999 to May 2011 and were treated for verrucous carcinoma of the oesophagus (table 1). Furthermore, the following single case report exemplifies local endoscopic therapy as a treatment strategy for verrucous carcinoma.

\section{Case Report}

A 77-year-old white man presented with the symptom of progressive odynophagia lasting for 6 months. External endoscopic examination raised suspicion of oesophageal cancer. Biopsies showed only chronic inflammatory changes. The patient was referred to our department for further diagnostic investigation in July 2007. There was no history of alcohol or tobacco use. When seen at our institution, endoscopy showed a $9 \mathrm{~cm}$ long, circumferential mucosal irregularity dominated by pavestone-like white plaques (fig. 1-4). Repetition of biopsy sampling and close communication with a specialized gastrointestinal pathologist led to the diagnosis

Table 1. Characteristics of the patients with oesophageal veruccous carcinoma

\begin{tabular}{llllll}
\hline Age, years & Sex & Symptoms & Risk factors & Tumor stage & Therapy \\
\hline 71 & M & odynophagia & none & pT1a(m2), L0, V0, G1 & LET (C) \\
67 & F & dysphagia & N, PD & pT1a(m1), L0, V0, G1 & LET (C) \\
48 & M & burning sensation & N, A, PD & pT1a(m1), L0, V0, G1 & LET (C) \\
76 & M & abdominal pain & none & pT1a(m1), L0, V0, R1, G1 & LET (C) \\
90 & F & odynophagia & N & pT1b(sm1), G1 & LET (P) \\
86 & M & dysphagia & A & uT1a(mx) & LET (P) \\
65 & M & burning sensation & N, A & pT1b(sm1), L0, V0, G1 & LET (P) \\
56 & M & dysphagia & N, A, PD & no data & LET (P) \\
74 & M & asymptomatic & none & uT2N0 & surgery (C) \\
73 & F & dysphagia & papillomatosis & pT1b(sm3), N0, G1 & surgery (C) \\
69 & F & asymptomatic & none & pT1N0, G1 & surgery (C) \\
70 & M & dysphagia & N, A & pT1a(m1)N0, G1 & surgery (C) \\
60 & M & dysphagia & N & uT3N1 & CRT (C) \\
67 & M & dysphagia & $\mathrm{N}$, A, PD & pT1b(smx), L0, V0, G1 & CRT (C) \\
57 & M & dysphagia & N & pTis & CRT (C) \\
\hline
\end{tabular}

${ }^{a}$ No data $=$ No staging examinations due to relevant comorbidities.

$\mathrm{A}=$ Alcohol abuse $; \mathrm{C}=$ curative CRT $=$ chemoradiotherapy LET $=$ local endoscopic therapy $\mathrm{m}=$ mucosal $; \mathrm{N}=$ nicotine; $\mathrm{P}=$ palliative $\mathrm{PD}=$ pseudodiverticulosis $; \mathrm{sm}=$ submucosal $\mathrm{x}=$ missing data. 


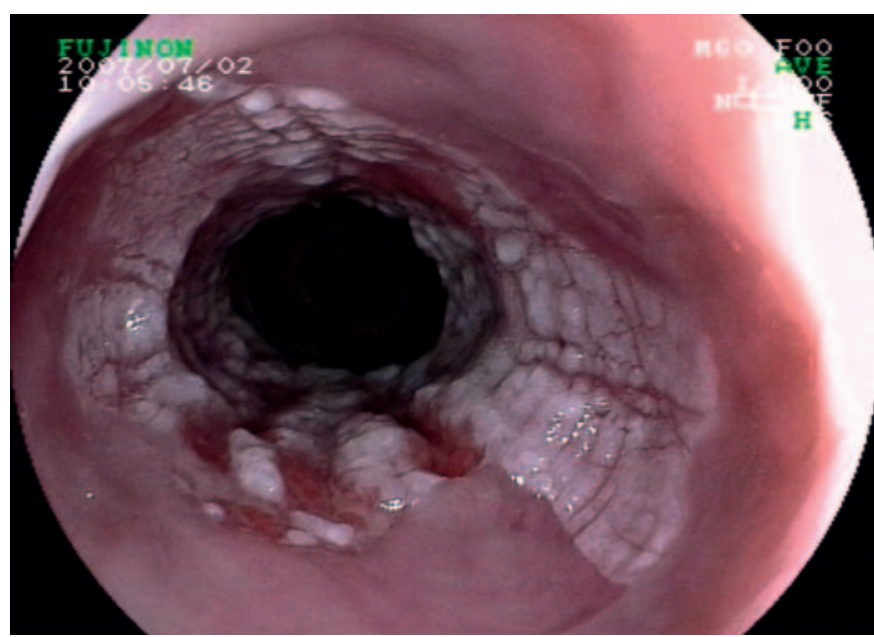

Fig. 1. Verrucous carcinoma and pseudodiverticulosis.

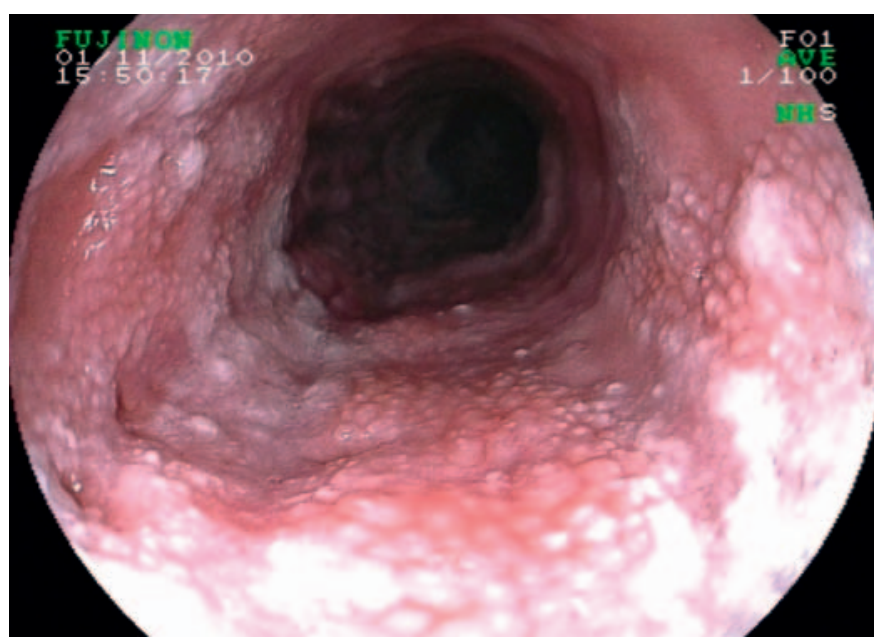

Fig. 2. Verrucous carcinoma.

of verrucous carcinoma. Endoscopic ultrasound, computed tomography, and abdominal ultrasound did not show any metastasis. The tumour was removed by local endoscopic resection in four endoscopic sessions. Histological assessment showed a mucosal carcinoma with no infiltration into lymph or blood vessels. Freedom from tumour at the basal margins was confirmed. Because of the extended superficial tumour growth, lateral margins of the resection specimen showed tumour infiltration $(\mathrm{pT} 1 \mathrm{a}(\mathrm{m} 2)$, V0, L0, G1, R1 (HM) R0 (VM)). Due to the extended procedures, the patients developed stenosis requiring bougienage. Follow-up examinations for the last 7 years showed no tumour recurrence.

\section{Results}

Oesophageal verrucous carcinomas were diagnosed in 15 patients during the mentioned period. The median age of the patients was 67 years (range 48-78 years) and the male-female sex ratio was $3: 1$. The HPV infection status was not proven due to the unknown aetiological role and more than $60 \mathrm{HPV}$ subtypes.

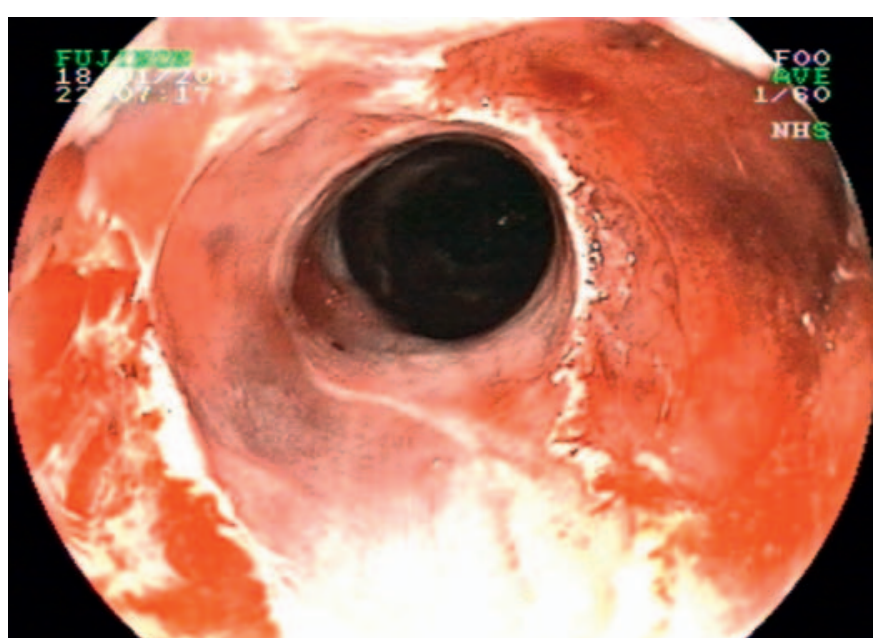

Fig. 3. Endoscopic image following multiple endoscopic resection in the patient shown in fig. 1 .

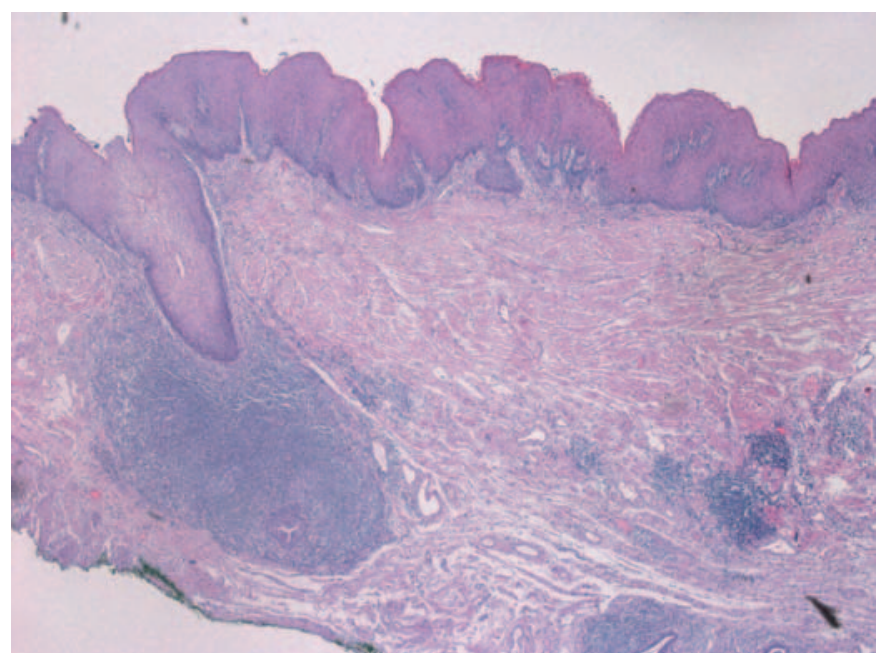

Fig. 4. Histological image of the same lesion.

\section{Symptoms and Risk Factors}

The cardinal symptom in 8 of the 15 patients was dysphagia, while 4 patients reported odynophagia. In 3 patients, the diagnosis was not based on oesophageal symptoms but was reached during diagnostic clarification of epigastric pain, while in 2 patients it was made in the context of preventive screening measures.

In 4 patients, the dysphagia was not caused by the tumour itself but by accompanying oesophageal diseases, particularly concomitant intramural pseudodiverticulosis. In addition, onethird of the patients (33\%) reported odynophagia. However, it was notable that the patients did not describe actual pain during the act of swallowing; all of them reported a burning sensation in the oesophagus during swallowing though. 3 of these 4 patients had concomitant reflux disease, which was documented either as endoscopically positive reflux disease or as Barrett's oesophagus. Conclusive differentiation between tumour symptoms and existing reflux symptoms is not possible. 
Table 2. Characteristics of patients and endoscopic findings

\begin{tabular}{ll}
\hline Characteristics & Result \\
\hline Median age & 67 years (range 48-78) \\
Men:women & $3: 1$ \\
Dysphagia/odynophagia & 12 \\
Nicotine/alcohol consumption & $10 / 7$ \\
Concomitant pseudodiverticulosis & 4 \\
Longitudinal extent of the tumor & $8 \mathrm{~cm}$ (range 2-22) \\
Tumor location (upper/upper-middle/ & $2 / 4 / 5 / 4$ \\
$\quad$ middle/lower part) & \\
uT1/T2/T3/no evaluation & $5 / 3 / 1 / 6$ \\
uN0/uN+/no evaluation & $11 / 3 / 1$ \\
\hline
\end{tabular}

As in classic oesophageal squamous cell carcinoma, patients in the present study with oesophageal verrucous carcinoma also had excess nicotine and alcohol consumption (64 and $49 \%$, respectively).

\section{Endoscopic Findings}

The endoscopic appearance of the tumour is typically that of a white, plaque-like surface raised slightly above the level of the mucosa (fig. 1). Only one of the tumours showed a polypoid growth pattern while all of the others were expanding superficially. The longitudinal extent of the tumours averaged $8 \mathrm{~cm}$ (range: $2-22 \mathrm{~cm}$ ). One tumour showed semicircular growth, but the majority (10 tumours) were circular in shape. There was no predominance for any typical location in the oesophagus in this cohort. 4 of the 15 patients were also diagnosed with intramural pseudodiverticulosis.

\section{Endosonographic Findings/Computed Tomography}

Endosonography was carried out in 14 of the 15 patients. In 1 patient, further diagnostic procedures were dispensed with, in view of severe comorbid conditions that took precedence clinically. The tumours were staged as uT1 in 5 of the 14 patients, as uT2 in 3 patients, and as uT3 in 1 other patient. In 5 patients, the tumour infiltration depth was not reported. Lymph node involvement was suspected on endosonography in 4 of these 14 patients (table 2).

\section{Treatment}

In accordance with the local endoscopic findings, the endosonographic diagnosis, and the existing comorbidities, the patients underwent local endoscopic therapy, radical oesophageal resection, or chemoradiotherapy. 9 of the 15 patients received endoscopic therapy, with curative intent in four cases and with palliative intent in five. Due to their comorbid condi- tions, the patients receiving palliative treatment were neither candidates for oesophageal resection nor for chemoradiotherapy. Oesophageal resection and chemoradiotherapy were carried out in 3 patients each.

In the group of 4 patients who received local endoscopic treatment with curative intent, endoscopic resections (1-48 interventions) were carried out, and in two cases additional thermal therapy was administered by means of potassium titanyl phosphate (KTP) laser treatment or argon plasma coagulation (APC) therapy. 2 of these patients are still receiving treatment (1 with one endoscopic resection and 1 with 48 endoscopic resections). A similar approach was used in the patients of the palliative endoscopy group. In the latter group, however, thermal therapy (0-7 endoscopic resections, APC treatment) more often took precedence over endoscopic resection.

Oesophageal resection was indicated in 3 patients. In 2 of these patients, this was because the endosonographic appearance of the tumour showed locally advanced stages (uT2N0, $\mathrm{uTxN} 1$ ). Surgery was recommended in the third patient as the oesophageal verrucous carcinoma had arisen against the background of papillomatosis which covered almost the entire oesophagus.

It was decided to carry out chemoradiotherapy in 3 patients. This was recommended for 2 of these patients because the upper margin of the tumour was at the upper oesophageal sphincter in both cases; one of these patients also had a status post partial gastric resection. The third patient had undergone hemicolectomy and partial liver resection 2 years prior to the diagnosis due to a colon carcinoma with hepatic metastasis; following a discussion with the patient, chemoradiotherapy was therefore preferable to oesophageal resection from our point of view.

\section{Comparison of Endosonographic Assessment with Histological Results}

Comparison of the endosonographic assessment with the histological results in the endoscopic and surgical resection specimens was possible in eight cases. Comparison was of course not possible in patients who underwent chemoradiotherapy on the basis of the endosonographic findings. In the group of patients who received endoscopic treatment, the endosonographic staging of uT1N0 corresponded to the specimen in 4 patients $(\mathrm{pT} 1 \mathrm{a}(\mathrm{m} 2), \mathrm{pT} 1 \mathrm{a}(\mathrm{m} 1), 2 \times \mathrm{pT} 1 \mathrm{~b}(\mathrm{sm}))$. In the group of patients who underwent surgery, by contrast, there was a marked discrepancy between the endosonographic assessment and the histological findings. 2 patients who were endosonographically assessed as having positive nodes were found to have no lymph node metastases in the surgical specimens (N0). The depth of infiltration of a tumour staged as UT2 was overestimated in 2 patients; histological analysis showed stage $\mathrm{T} 1 \mathrm{a}(\mathrm{m})$. 


\section{Treatment Results}

\section{Endoscopic Resection}

In the group of 4 patients who received endoscopic treatment with curative intent, 2 patients are currently still undergoing treatment. One of the patients had a remission of the tumour for 2.5 years and then died of a heart attack, and another patient has been in remission for 3 months.

Of the 5 patients who received palliative endoscopic treatment, one is still undergoing therapy, 2 patients have been in remission for 14 and 32 months, respectively, and 1 patient died of the tumour just under 5 years after the diagnosis. 1 patient failed to attend check-up appointments and could not be contacted. 3 of the endoscopically treated patients developed stenosis requiring bougienage following treatment.

\section{Oesophageal Resection}

Two of the 3 patients are currently in remission. 1 patient died postoperatively due to septic sequelae of an anastomotic insufficiency.

\section{Combined Chemoradiotherapy}

Of the 3 patients who underwent chemoradiotherapy (60 Gy/cisplatin, 5-fluorouracil), one is currently still receiving treatment, another has been in remission for 18 months, and the third has withdrawn from follow-up and cannot be contacted. 1 patient in the chemoradiotherapy group developed a post-therapeutic stenosis that required bougienage.

\section{Discussion}

The present report is the largest study to have been published worldwide on the entity of verrucous carcinoma of the oesophagus. It describes the essential characteristics of this type of tumour. Following the publication of a single case report [9], it is the first study which describes the option of local endoscopic therapy and the results in a total of 9 patients, with the procedure being carried out in 4 of whom with curative intent.

Due to the lack of evidence available for the treatment of verrucous carcinoma, treatment has so far been carried out in analogy with ordinary squamous cell carcinomas of the oesophagus. The present case series impressively shows that there are fundamental differences between the two tumour entities and that a direct transfer of the therapeutic strategies does not appear to be appropriate. The study suggests that the treatment approaches established for ordinary cases of oesophageal squamous cell carcinoma should not be blindly applied to patients with oesophageal verrucous carcinomas. Instead, individualized treatment approaches should be discussed with the patients. It should be noted in qualification that this survey represents a retrospective analysis including only a small number of cases, with a total of 15 patients.
Major differences between squamous cell carcinoma and verrucous carcinoma of the oesophagus are the oesophageal growth pattern of the tumour, the frequency of lymph node and distant metastases, and the response rate to chemoradiotherapy.

In the majority of patients, the growth pattern in oesophageal verrucous carcinomas is one of extensive superficial expansion (with a mean longitudinal tumour extension of $8 \mathrm{~cm}$, usually with circular growth). Stage T1 was present in 11 of 12 patients, despite the large size of the lesions. The 3 patients who received chemoradiotherapy are not taken into account here, as histological data on the surgical specimens were of course not available for these patients.

In four of the 15 cases, a secondary diagnosis of intramural pseudodiverticulosis was made. A study by Plavsic et al. [10] showed that squamous cell carcinoma of the oesophagus occurs significantly more often when intramural pseudodiverticulosis is present. Thus, possible neoplastic changes also have to be taken into account in the differential diagnosis of any stricture. The present study describes the first 4 patients with coexistent verrucous carcinoma and intramural pseudodiverticulosis.

The second main difference between verrucous carcinoma and squamous cell carcinoma of the oesophagus is the frequency of lymph node and distant metastases. Lymph node metastases were reported in 2 of 23 patients described in the literature. The 2 patients affected had locally very advanced tumours (T4). Distant metastasis has not been reported in any of the cases. Consistent with this finding, none of the patients in the present case series had a confirmed lymph node metastasis, and none had any distant metastases. In the group of patients who received endoscopic therapy, none of the patients in this series, even in the palliative group, was found to have any evidence of lymph node involvement. Attention should be drawn to 3 patients with tumours that showed infiltration of the upper submucosa (pT1b(sm1)). Over follow-up periods of 32 and 60 months, respectively, 2 of these patients did not develop any evidence of lymphatic metastases. 1 patient is currently still receiving treatment.

The surgical specimen showed no lymphatic metastases in the 2 patients in this study who underwent surgery and in whom an N1 stage had been suspected on endosonography. It is therefore also doubtful whether 2 patients whose endosonographic assessment showed node-positive findings and who underwent chemoradiotherapy actually had metastases. Appropriately, the series from Rochester, published as an abstract so far, has also pointed out an overstaging by endoscopic ultrasound [8]. The histological analysis of lymph nodes in surgical specimens described in the literature reports a finetissue picture of lymphadenitis, with histiocytic, hyperplastic, or reactive changes in some of the patients [3]. These changes may lead to an overassessment of the lymph node status.

In comparison to these data, a recently published Japanese study reported a lymph node metastasis rate of $31 \%$ among 145 patients with pT1 oesophageal squamous cell cancer [11]. 
The third main difference between the two tumour entities is seen in the response to chemoradiotherapy. The effectiveness of radiation in verrucous carcinoma of the oesophagus must be categorised as much lower in comparison with oesophageal squamous cell carcinoma. If the results of radiotherapeutic treatment for verrucous carcinomas in the ENT field can be used for comparison, a local control rate of only $43 \%$ can be expected (64/148 patients). The anaplastic transformations of verrucous carcinomas that have been reported on several occasions following radiotherapy have various causes and can only be expected in a small percentage of the cases $(7 \% ; 6 / 148)$. However, they appear to be associated with a poor prognosis [12].

The superficial growth pattern, very low rate of lymph node metastases, and absence of any reported distant metastases from verrucous carcinomas of the oesophagus make local endoscopic therapy appear appropriate for this tumour entity. Although only 9 patients in the present study received endoscopic therapy, it becomes clear by analogy with the treatment of early squamous cell carcinoma of the oesophagus and of Barrett's adenocarcinoma that endoscopic resection even of extensive areas is technically possible and safe for the patient [13]. In view of the extensiveness of the resection that is often needed for verrucous carcinoma of the oesophagus, patients have to be informed about the risk of post-therapeutic stenosis, as the percentage is in the double-digit range after extensive resections [14, 15]. Endoscopic submucosal dissection with removal of the entire tumour is the endoscopic technique that should in principle be aimed for. As a result of intramural inflammatory processes such as those that can be caused by pseudodiverticulosis, for example, endoscopic resection may not be possible. An alternative is to carry out multiple 'classic' endoscopic resections using the cap or ligation technique, as in the present study. In comparison with oesophageal resection, there is almost no risk of severe complications associated with endoscopic therapy. No treatment-related fatalities have occurred in our experience in more than 1,000 patients treated [13-16]. Even when there is infiltration of the deep mucosa $(\mathrm{m} 3)$ in ordinary squamous cell carcinoma, oesophageal resection is considered worth discussing. Local endoscopic therapy in the presence of oesophageal verrucous carcinoma, in analogy with the indications for local en- doscopic therapy for Barrett's adenocarcinoma (e.g. even up to $\mathrm{pT} 1 \mathrm{~b}(\mathrm{sm} 1))$, is therefore conceivable - although the data are extremely limited.

In summary, the notably different characteristics of verrucous carcinoma of the oesophagus in comparison with oesophageal squamous cell carcinoma raise doubts as to whether the treatment recommendations for squamous cell carcinoma can be transferred to this entity on a one-to-one basis. In addition, critical attention must be paid to the endosonographic findings, as the present analysis of data shows that these tend to lead to overstaging.

If a locally advanced tumour stage $(\mathrm{uTxN}+)$ is found at the initial diagnosis and surgery is technically possible, then - in view of the probable rarity of lymph node metastases and the limited radiosensitivity of the tumour - we would in principle prefer an oesophageal resection to chemoradiotherapy in suitable patients. The presence of concomitant oesophageal diseases also needs to be taken into account. If coexistent pseudodiverticulosis is present, this would be a further argument in favour of oesophageal resection.

Due to the rarity of this entity, the case numbers are unfortunately so limited that evidence-based recommendations are unlikely to become available even in the future as a result of a lack of studies and long-term data.

In our view, it is in principle possible even in tumours with very extensive superficial growth to carry out a diagnostic endoscopic resection in order to allow histological confirmation of the tumour stage. The therapeutic approach can then be based on the results. In view of the usually extensive endoscopic interventions that are needed for resection of the often large tumour surfaces, as well the technical difficulty of endoscopic resection that sometimes results from intramural inflammatory processes, such patients should be referred to a centre with expertise in local endoscopic treatment. It can be assumed that curative local endoscopic treatment is possible in patients with early verrucous carcinomas of the oesophagus.

\section{Disclosure Statement}

This study was not supported in the form of grants, equipment, or pharmaceuticals.

\section{References}

1 Montgomery E, Field JK, Boffeta P, et al: Squamous cell carcinoma of the esophagus; in Bosman FT, Carneiro F, Hruban RH, et al. (eds): WHO Classification of Tumours of the Digestive System, ed 4. Lyon, International Agency for Research on Cancer, 2010.

2 Kavin H, Yaremko L, Valaitis J, et al: Chronic esophagitis evolving to verrucous squamous cell carcinoma: possible role of exogenous chemical carcinogens. Gastroenterology 1996;110:904-914.
3 Saghravanian N, Ghazvini K, Babakoohi S, et al: Low prevalence of high risk genotypes of human papilloma virus in normal oral mucosa, oral leukoplakia and verrucous carcinoma. Acta Odontol Scand 2011,69:406-409.

4 Osborn NK, Keate RF, Trastek VF, et al: Verrucous carcinoma of the esophagus: clinicopathophysiologic features and treatment of a rare entity. Dig Dis Sci 2003;48:465-474.
5 Kraus FT, Perezmesa C: Verrucous carcinoma. Clinical and pathologic study of 105 cases involving oral cavity, larynx and genitalia. Cancer 1966;19: 26-38.

6 Terada T: Verrucous carcinoma of the skin: a report on 5 Japanese cases. Ann Diagn Pathol 2011; 15:175-180.

7 Minielly JA, Harrison EG Jr, Fontana RS, et al: Verrucous squamous cell carcinoma of the esophagus. Cancer 1967;20:2078-2087. 
8 Sweetser S, Jacobs NL, Song LM: Endoscopic diagnosis and treatment of esophageal verrucous squamous cell cancer. Dis Esophagus 2012;27:452-456.

9 Tajiri H, Muto M, Boku N, et al: Verrucous carcinoma of the esophagus completely resected by endoscopy. Am J Gastroenterol 2000;95:1076-1077.

10 Plavsic BM, Chen MY, Gelfand DW, et al: Intramural pseudodiverticulosis of the esophagus detected on barium esophagograms: increased prevalence in patients with esophageal carcinoma. AJR Am J Roentgenol 1995;165:1381-1385.

11 Tanaka T, Matono S, Mori N: T1 squamous cell carcinoma of the esophagus: long-term outcomes and prognostic factors after esophagectomy. Ann Surg Oncol 2014;21:932-938.
12 Ferlito A, Rinaldo A, Mannarà GM: Is primary radiotherapy an appropriate option for the treatment of verrucous carcinoma of the head and neck? J Laryngol Otol 1998;112:132-139.

13 Pech O, Behrens A, May A, et al: Long-term results and risk factor analysis for recurrence after curative endoscopic therapy in 349 patients with high-grade intraepithelial neoplasia and mucosal adenocarcinoma in Barrett's oesophagus. Gut 2008;57:1200-1206.
14 Pech O, May A, Gossner L, et al: Curative endoscopic therapy in patients with early esophageal squamous-cell carcinoma or high-grade intraepithelial neoplasia. Endoscopy 2007;39:30-35.

15 Pech O, Gossner L, May A, et al: Endoscopic resection of superficial esophageal squamous-cel carcinomas: Western experience. Am J Gastroenterol 2004;99:1226-1232.

16 Pech O, May A, Manner H, et al: Long-term efficacy and safety of endoscopic resection for patients with mucosal adenocarcinoma of the esophagus. Gastroenterology 2014;146:652-660. 